\title{
OVERLAPPING ADDITIVE SCHWARZ PRECONDITIONERS FOR ELLIPTIC PDES ON THE UNIT SPHERE
}

\author{
Q. T. LE GIA, I. H. SLOAN, AND T. TRAN
}

\begin{abstract}
We present an overlapping domain decomposition technique for solving elliptic partial differential equations on the sphere. The approximate solution is constructed using shifts of a strictly positive definite kernel on the sphere. The condition number of the Schwarz operator depends on the way we decompose the scattered set into smaller subsets. The method is illustrated by numerical experiments on relatively large scattered point sets taken from MAGSAT satellite data.
\end{abstract}

\section{INTRODUCTION}

Partial differential equations on the sphere have many applications, for example in weather forecasting models and geophysics. In this paper we consider an elliptic equation on the unit sphere of the form

$$
-\Delta^{*} u+\omega^{2} u=f
$$

where $\Delta^{*}$ is the Laplace-Beltrami operator and $\omega$ is some nonzero real constant. This elliptic equation arises, for example, when one discretizes in time the diffusion equation on the sphere.

When solving elliptic PDEs on the unit sphere based on scattered measured data, with the approximate solution constructed using shifts of a strictly positive definite kernel on the sphere, a very ill-conditioned linear system results, whether a Galerkin method [8] or a collocation method [13] is used. This is due to the separation radius of the scattered data [11, which can be very small for a large set of scattered data.

In this paper, we propose a way of partitioning a given scattered data set into smaller subsets. This method of partitioning is based on the property that data sites along the track of a satellite form a sequence of discrete points, see Figure 1, and this sequence covers the globe (except for two small polar caps, in this case of radius about 0.1 radian) over a period of time. This happens because the Earth is rotating around its own axis, while the satellite traverses from near the North pole to near the South pole then back to the North pole in an elliptical path.

Based on those overlapping subsets of scattered data, we define an additive Schwarz operator for solving (11). We prove a theorem which gives a bound on the condition number of the Schwarz operator. The method is illustrated by numerical

Received by the editor December 15, 2006 and, in revised form, January 31, 2008.

2000 Mathematics Subject Classification. Primary 33F05, 65N55; Secondary 65N30.

Key words and phrases. Sphere, spherical basis function, domain decomposition, elliptic partial differential equation. 
experiments on relatively large scattered point sets taken from MAGSAT satellite data (see [10]).

It is noted that for the interpolation problem in $\mathbb{R}^{n}$ using radial basis functions, the idea of dividing the scattered data set into smaller subsets for the purpose of defining the Schwarz alternating algorithm has been proposed in 1]. However, in that paper it is proved only that the Schwarz alternating method is a contraction. Moreover, the method is not used there as a preconditioner, and the problem to which it is applied, namely interpolation with thin plate splines in $\mathbb{R}^{n}$, is different from that studied here.

Work on applying the multiplicative Schwarz alternating algorithm using spherical splines has also been carried out in [5, but in that work the data points are not scattered, and again the Schwarz method is not used as a preconditioner.

The structure of the paper is as follows. In Section 2, we will review spherical harmonics and Sobolev spaces on the unit sphere. Then, in Section 3, the elliptic partial differential equation is presented. The abstract framework for the additive Schwarz preconditioner is reviewed in Section 4. In Section 5, we present the main theoretical results of the paper. The final two sections describe the algorithm and present numerical results based on real scattered data. In the paper, generic constants are denoted by $c, c_{1}, c_{2}, c_{3} \ldots$

\section{Preliminaries}

In this section, we will review spherical harmonics, function spaces on the unit Euclidean sphere $S^{n} \subset \mathbb{R}^{n+1}$, and spherical basis functions.

2.1. Spherical harmonics. Spherical harmonics are the restriction of homogeneous harmonic polynomials in $\mathbb{R}^{n+1}$ to the unit sphere $S^{n}$. We denote an orthonormal (with respect to the $L^{2}\left(S^{n}\right)$ inner product) basis for the spherical harmonics of degree $\ell$ by

$$
\left\{Y_{\ell, k}: k=1, \ldots, N(n, \ell)\right\}, \quad \ell=0,1, \ldots,
$$

where $N(n, \ell)$ is the dimension of the space of all spherical harmonics of degree $\ell$; the values of $N(n, \ell)$ are (see [14]):

$$
N(n, 0)=1 \text { and } N(n, \ell)=\frac{(2 \ell+n-1) \Gamma(\ell+n-1)}{\Gamma(\ell+1) \Gamma(n)} \text { for } \ell \geq 1 .
$$

The asymptotic behavior of $N(n, \ell)$ for fixed $n$ and increasing $\ell$ is $O\left(\ell^{n-1}\right)$. The spherical harmonics $\left\{Y_{\ell, k}: \ell=0,1, \ldots ; k=1, \ldots, N(n, \ell)\right\}$ form a complete orthonormal basis for $L^{2}\left(S^{n}\right)$. Correspondingly, for a given function $f \in L^{2}\left(S^{n}\right)$, we define its Fourier coefficients by

$$
\widehat{f}_{\ell, k}=\int_{S^{n}} f(x) Y_{\ell, k}(x) d S(x),
$$

where $d S$ is the surface measure of the sphere $S^{n}$, and represent $f$ as a Fourier series,

$$
f=\sum_{\ell=0}^{\infty} \sum_{k=1}^{N(n, \ell)} \widehat{f}_{\ell, k} Y_{\ell, k},
$$

in which the equals sign is understood in the $L^{2}\left(S^{n}\right)$ sense. 
Spherical harmonics of degree $\ell$ are eigenfunctions of the Laplace-Beltrami operator $\Delta^{*}$ on $S^{n}$, with eigenvalues $-\lambda_{\ell}$, with

$$
\lambda_{\ell}=\ell(\ell+n-1)
$$

i.e.,

$$
\Delta^{*} Y_{\ell, k}=-\lambda_{\ell} Y_{\ell, k} .
$$

The addition formula for spherical harmonics of the same degree $\ell$ (see [14]) is

$$
\sum_{k=0}^{N(n, \ell)} Y_{\ell, k}(x) Y_{\ell, k}(y)=\frac{1}{\omega_{n}} N(n, \ell) P_{\ell}(n+1 ; x \cdot y),
$$

where $P_{\ell}(n+1 ; t)$ is the normalized Legendre polynomial of degree $\ell$ in $\mathbb{R}^{n+1}$ and $\omega_{n}$ is the surface area of the unit sphere $S^{n}$. Recall from [14] that $P_{\ell}(n+1 ; 1)=1$ and

$$
\int_{-1}^{+1} P_{\ell}(n+1 ; t) P_{k}(n+1 ; t)\left(1-t^{2}\right)^{(n-2) / 2} d t=\frac{\omega_{n}}{\omega_{n-1} N(n, \ell)} \delta_{\ell, k},
$$

where $\omega_{n-1}$ is the surface area of the sphere $S^{n-1}$, and $\delta_{\ell, k}$ is the Kronecker delta.

For a given $s \geq 0$, the Sobolev space $H^{s}\left(S^{n}\right)$ on the unit sphere is defined in terms of the eigenvalues of the Laplace-Beltrami operator (see [12]),

$$
H^{s}\left(S^{n}\right):=\left\{f \in L^{2}\left(S^{n}\right): \sum_{\ell=0}^{\infty} \sum_{k=1}^{N(n, \ell)}\left(1+\lambda_{\ell}\right)^{s}\left|\widehat{f}_{\ell, k}\right|^{2}<\infty\right\} .
$$

The norm of a function $f$ in this space is defined to be

$$
\|f\|_{H^{s}\left(S^{n}\right)}=\left(\sum_{\ell=0}^{\infty} \sum_{k=1}^{N(n, \ell)}\left(1+\lambda_{\ell}\right)^{s}\left|\widehat{f}_{\ell, k}\right|^{2}\right)^{1 / 2} .
$$

2.2. Sobolev spaces on the sphere through a specific atlas. Sobolev spaces on $S^{n}$ can also be defined using local charts (see [12]). Here we use a specific atlas of charts, as in 6 .

Let a spherical cap of radius $\alpha$ centered at $p \in S^{n}$ be defined by

$$
C(p, \alpha):=\left\{x \in S^{n}: \theta(p, x) \leq \alpha\right\},
$$

where $\theta(p, x)=\cos ^{-1}(p \cdot x)$ is the geodesic distance between two points $x, p \in S^{n}$. The interior of $C(p, \alpha)$ is denoted by $C^{o}(p, \alpha)$. Let $\hat{n}=(0, \ldots, 0,1)$ and $\hat{s}=$ $(0, \ldots, 0,-1)$ denote the north and south poles of $S^{n}$, respectively. Then a simple cover for the sphere is provided by

$$
U_{1}=C^{o}\left(\hat{n}, \theta_{0}\right) \quad \text { and } \quad U_{2}=C^{o}\left(\hat{s}, \theta_{0}\right) \text {, where } \theta_{0} \in(\pi / 2,2 \pi / 3) .
$$

The stereographic projection $\sigma_{\hat{n}}$ of the punctured sphere $S^{n} \backslash\{\hat{n}\}$ onto $\mathbb{R}^{n}$ is defined as a mapping that maps $x \in S^{n} \backslash\{\hat{n}\}$ to the intersection of the equatorial hyperplane $\{z=0\}$ and the extended line that passes through $x$ and $\hat{n}$. The stereographic projection $\sigma_{\hat{s}}$ based on $\hat{s}$ can be defined analogously. We set

$$
\psi_{1}=\left.\frac{1}{\tan \left(\theta_{0} / 2\right)} \sigma_{\hat{s}}\right|_{U_{1}} \quad \text { and } \quad \psi_{2}=\left.\frac{1}{\tan \left(\theta_{0} / 2\right)} \sigma_{\hat{n}}\right|_{U_{2}},
$$

so that $\psi_{k}, k=1,2$, maps $U_{k}$ onto $B(0,1)$, the unit ball in $\mathbb{R}^{n}$. We conclude that $\mathcal{A}=\left\{U_{k}, \psi_{k}\right\}_{k=1}^{2}$ is a $C^{\infty}$ atlas of covering coordinate charts for the sphere. It is known (see [19]) that the stereographic coordinate charts $\left\{\psi_{k}\right\}_{k=1}^{2}$ as defined in (8) 
map spherical caps to Euclidean balls, but in general, concentric spherical caps are not mapped to concentric Euclidean balls. The projection $\psi_{k}$, for $k=1,2$, does not distort too much the geodesic distance between two points $x, y \in S^{n}$, as shown in 9 .

With the atlas so defined, we define the map $\pi_{k}$ which takes a real-valued function $g$ with compact support in $U_{k}$ into a real-valued function on $\mathbb{R}^{n}$ by

$$
\pi_{k}(g)(x)= \begin{cases}g \circ \psi_{k}^{-1}(x), & \text { if } x \in B(0,1) \\ 0, & \text { otherwise. }\end{cases}
$$

Let $\left\{\chi_{k}: S^{n} \rightarrow \mathbb{R}\right\}_{k=1}^{2}$ be a partition of unity subordinate to the atlas, i.e., a pair of nonnegative infinitely differentiable functions $\chi_{k}$ on $S^{n}$ with compact support in $U_{k}$ such that $\sum_{k} \chi_{k}=1$. For any function $f: S^{n} \rightarrow \mathbb{R}$, we can use the partition of unity to write

$$
f=\sum_{k=1}^{2}\left(\chi_{k} f\right), \text { where }\left(\chi_{k} f\right)(p)=\chi_{k}(p) f(p), \quad p \in S^{n} .
$$

The Sobolev space $H^{1}\left(S^{n}\right)$ is defined to be the set

$$
\left\{f \in L^{2}\left(S^{n}\right): \pi_{k}\left(\chi_{k} f\right) \in H^{1}\left(\mathbb{R}^{n}\right) \quad \text { for } k=1,2\right\},
$$

which is equipped with the norm

$$
\|f\|_{H^{1}\left(S^{n}\right)}=\left(\sum_{k=1}^{2}\left\|\pi_{k}\left(\chi_{k} f\right)\right\|_{H^{1}\left(\mathbb{R}^{n}\right)}^{2}\right)^{1 / 2}
$$

This $H^{1}\left(S^{n}\right)$ norm is equivalent to the $H^{1}\left(S^{n}\right)$ norm given in Section 2.1 (see [12]).

2.3. Spherical basis functions. In this section, we will review the necessary background on positive definite kernels on the unit sphere and spherical basis functions.

A real-valued kernel $\Phi$ in $C\left(S^{n} \times S^{n}\right)$ is termed positive definite on $S^{n}$ if $\Phi(x, y)=$ $\Phi(y, x)$ and if for every finite set of distinct points $X=\left\{x_{1}, \ldots, x_{N}\right\}$ on $S^{n}$, the symmetric $N \times N$ matrix $A$ with entries $A_{i, j}=\Phi\left(x_{i}, x_{j}\right)$ is positive semi-definite. If the matrix $A$ is positive definite, then $\Phi$ is called a strictly positive definite kernel (see [20, 31]).

Let $\phi$ be a univariate function defined on $[-1,1]$ which can be expanded in terms of Legendre polynomials as

$$
\phi(t)=\frac{1}{\omega_{n}} \sum_{\ell=0}^{\infty} N(n, \ell) \widehat{\phi}(\ell) P_{\ell}(n+1 ; t),
$$

where

$$
\widehat{\phi}(\ell)=\omega_{n-1} \int_{-1}^{+1} \phi(t) P_{\ell}(n+1 ; t)\left(1-t^{2}\right)^{(n-2) / 2} d t .
$$

Due to the addition formula (3), a kernel $\Phi$ defined by

$$
\Phi(x, y)=\phi(x \cdot y)
$$

can be represented as

$$
\Phi(x, y)=\sum_{\ell=0}^{\infty} \sum_{k=1}^{N(n, \ell)} \widehat{\phi}(\ell) Y_{\ell, k}(x) Y_{\ell, k}(y) .
$$


In [3, a complete characterization of strictly positive definite kernels is established: the kernel $\Phi$ is strictly positive definite if and only if $\widehat{\phi}(\ell) \geq 0$ for all $\ell \geq 0$ and $\widehat{\phi}(\ell)>0$ for infinitely many even values of $\ell$ and infinitely many odd values of $\ell$; see also 20 and 31 .

The native space $\mathcal{N}_{\Phi}$ associated with the kernel $\Phi$ is defined by

$$
\mathcal{N}_{\Phi}:=\left\{f \in L^{2}\left(S^{n}\right): \sum_{\ell=0}^{\infty} \sum_{k=1}^{N(n, \ell)} \frac{\left|\widehat{f}_{\ell, k}\right|^{2}}{\widehat{\phi}(\ell)}<\infty\right\} .
$$

If

$$
c_{1}\left(1+\lambda_{\ell}\right)^{-s} \leq \widehat{\phi}(\ell) \leq c_{2}\left(1+\lambda_{\ell}\right)^{-s},
$$

where $c_{1}, c_{2}>0$ are some constants and $s>n / 2$, the native space $\mathcal{N}_{\Phi}$ can be identified with the Sobolev space $H^{s}\left(S^{n}\right)$ defined in (5). Henceforth, the condition (15) is shortened to $\widehat{\phi}(\ell) \sim\left(1+\lambda_{\ell}\right)^{-s}$.

In the following we define a positive definite kernel $\Phi$ from a univariate function $\phi$ satisfying (15) by using Wendland's compactly supported radial basis functions [27. For any nonnegative integer $j$, let

$$
\rho_{j}(r)= \begin{cases}(1-r)^{j}, & 0<r \leq 1 \\ 0, & r>1\end{cases}
$$

and let

$$
I \rho_{j}(r)=\int_{r}^{\infty} s \rho_{j}(s) d s, \quad r \geq 0 .
$$

We define, for any nonnegative integer $m$,

$$
\rho_{n+1, m}(r)=I^{m} \rho_{m+\left\lfloor\frac{n+1}{2}\right\rfloor+1}(r) .
$$

The radial basis function $\Psi_{n+1, m}$ is defined in $\mathbb{R}^{n+1}$ as

$$
\Psi_{n+1, m}(x)=\rho_{n+1, m}(\|x\|),
$$

where $\|\cdot\|$ denotes the Euclidean norm in $\mathbb{R}^{n+1}$. It is shown in 28, Corollary 2.3] that $\Psi_{n+1, m} \in C^{2 m}\left(\mathbb{R}^{n+1}\right)$. For any given $N$ and any set of $N$ pairwise distinct points $\left\{x_{1}, \ldots, x_{N}\right\}$ in $\mathbb{R}^{n+1}$, the matrix

$$
\left[\Psi_{n+1, m}\left(x_{i}-x_{j}\right)\right]_{i, j=1}^{N}
$$

is positive definite; see [29, Theorem 9.13]. Since $\|x-y\|=\sqrt{2-2 x \cdot y}$ for any $x, y \in S^{n}$, the kernel $\Phi$ defined by (12) with

$$
\phi(t)=\rho_{n+1, m}(\sqrt{2-2 t})
$$

is related to the above radial basis function $\Psi_{n+1, m}$ by

$$
\Phi(x, y)=\Psi_{n+1, m}(x-y), \quad x, y \in S^{n} .
$$

Since the matrix (16) is positive definite, $\Phi$ is a strictly positive definite kernel on the sphere $S^{n}$. Moreover, the asymptotic behavior of the Fourier coefficients $\widehat{\phi}(\ell)$ is, see [15, Proposition 4.6],

$$
\widehat{\phi}(\ell)=O\left(\ell^{-2 m-n-1}\right) .
$$

Using (2), we deduce that $\phi$ satisfies (15) with

$$
s=m+(n+1) / 2 .
$$


With this kernel $\Phi$, we can now establish a set of spherical basis functions (SBFs) $\left\{\Phi_{1}, \ldots, \Phi_{N}\right\}$ associated with a set $X=\left\{x_{1}, \ldots, x_{N}\right\}$ of scattered and distinct points on $S^{n}$, where

$$
\Phi_{i}(x):=\Phi\left(x_{i}, x\right) .
$$

The finite-dimensional space spanned by these SBFs is denoted by $V_{X}$ :

$$
V_{X}:=\operatorname{span}\left\{\Phi_{i}: i=1, \ldots, N\right\} .
$$

We note that the SBFs $\Phi_{i}, i=1, \ldots, N$, depend only on the geodesic distance between the points $x$ and $x_{i}$. The set $X$ is characterized by its mesh norm $h_{X}$ and separation radius $q_{X}$, defined by

$$
h_{X}:=\sup _{y \in S^{n}} \min _{x_{i} \in X} \theta\left(x_{i}, y\right) \quad \text { and } \quad q_{X}:=\frac{1}{2} \min _{i \neq j} \theta\left(x_{i}, x_{j}\right) .
$$

The kernel $\Phi$ being strictly positive definite, the interpolation problem in $V_{X}$ of scattered points using spherical basis functions is always solvable. Given a function $f$ whose values $f\left(x_{j}\right)$ for $j=1, \ldots, N$ are known, the interpolant $I_{X} f$ of $f$ is defined as a linear combination of the SBFs which satisfies $\left(I_{X} f\right)\left(x_{j}\right)=f\left(x_{j}\right)$ for all $j=1, \ldots, N$. It is observed in [11] that the matrix $A$ with entries $A_{i, j}=\Phi_{i}\left(x_{j}\right)$ arising from this interpolation problem is ill-conditioned. More fully, it is shown there that the least eigenvalue of the matrix $A$ depends on the separation radius $q_{X}$ of the set $X$, which can be very small for a large set of scattered data, and also on the smoothness of the kernel $\Phi$. (The smoother the kernel the smaller the least eigenvalue of $A$.)

\section{The ELLIPTIC PARTIAL DifFERENTIAL EQUATION ON SPHERES}

We consider the following elliptic partial differential equation on the unit sphere:

$$
-\Delta^{*} u+\omega^{2} u=f,
$$

where $f$ is some given function and $\omega \neq 0$ is a real constant. This type of elliptic partial differential equations arises when one discretizes in time the heat equation on the surface of the sphere. To set up a weak formulation, we introduce the bilinear form

$$
a(u, v):=\sum_{\ell=0}^{\infty} \sum_{k=1}^{N(n, \ell)}\left(\lambda_{\ell}+\omega^{2}\right) \widehat{u}_{\ell, k} \widehat{v}_{\ell, k} .
$$

A natural weak formulation of the elliptic PDE is

$$
a(u, v)=\langle f, v\rangle \quad \forall v \in H^{1}\left(S^{n}\right),
$$

where $\langle u, v\rangle=\int_{S^{n}} u v d S$. The bilinear form is bounded and coercive by the following lemma.

Lemma 3.1. For $u, v \in H^{1}\left(S^{n}\right)$, there are positive constants $A_{0} \leq 1$ and $A_{1} \geq 1$ such that

$$
a(u, v) \leq A_{1}\|u\|_{H^{1}\left(S^{n}\right)}\|v\|_{H^{1}\left(S^{n}\right)} \text { and } a(u, u) \geq A_{0}\|u\|_{H^{1}\left(S^{n}\right)}^{2} .
$$


Proof. In terms of Fourier series, we have

$$
\begin{aligned}
a(u, v) & =\sum_{\ell=0}^{\infty} \sum_{k=1}^{N(n, \ell)}\left(\lambda_{\ell}+\omega^{2}\right) \widehat{u}_{\ell, k} \widehat{v}_{\ell, k} \\
& \leq \max \left\{1, \omega^{2}\right\} \sum_{\ell=0}^{\infty} \sum_{k=1}^{N(n, \ell)}\left(\lambda_{\ell}+1\right) \widehat{u}_{\ell, k} \widehat{v}_{\ell, k} \\
& \leq A_{1}\left(\sum_{\ell=0}^{\infty} \sum_{k=1}^{N(n, \ell)}\left(\lambda_{\ell}+1\right)\left|\widehat{u}_{\ell, k}\right|^{2}\right)^{1 / 2}\left(\sum_{\ell=0}^{\infty} \sum_{k=1}^{N(n, \ell)}\left(\lambda_{\ell}+1\right)\left|\widehat{v}_{\ell, k}\right|^{2}\right)^{1 / 2} \\
& =A_{1}\|u\|_{H^{1}}\|v\|_{H^{1}},
\end{aligned}
$$

where $A_{1}=\max \left\{1, \omega^{2}\right\}$. For the second inequality, we have

$$
\begin{aligned}
a(u, u) & =\sum_{\ell=0}^{\infty} \sum_{k=1}^{N(n, \ell)}\left(\lambda_{\ell}+\omega^{2}\right)\left|\widehat{u}_{\ell, k}\right|^{2} \\
& \geq \min \left\{1, \omega^{2}\right\} \sum_{\ell=0}^{\infty} \sum_{k=1}^{N(n, \ell)}\left(\lambda_{\ell}+1\right)\left|\widehat{u}_{\ell, k}\right|^{2} \\
& =A_{0}\|u\|_{H^{1}\left(S^{n}\right)}^{2},
\end{aligned}
$$

where $A_{0}=\min \left\{1, \omega^{2}\right\}$.

We remark that a direct consequence of the lemma is that $a(u, u) \sim\|u\|_{H^{1}\left(S^{n}\right)}^{2}$. The Ritz-Galerkin approximation problem is:

$$
\text { Find } u_{X} \in V_{X} \text { such that } a\left(u_{X}, v\right)=\langle f, v\rangle \quad \forall v \in V_{X} .
$$

The problem will reduce to the problem of solving the following linear system:

$$
B \mathbf{c}=\mathbf{f},
$$

where the entries of the matrix $\boldsymbol{B}$ are given as $B_{i, j}=a\left(\Phi_{i}, \Phi_{j}\right)$ and the vector $\mathbf{f}$ is given as $\mathbf{f}=\left[f_{j}\right]_{j=1}^{N}$ in which $f_{j}=\left\langle f, \Phi_{j}\right\rangle$ for $i, j=1, \ldots, N$.

The following lemma, which is a variant of the Funk-Hecke formula (see [14]), facilitates the computation of each entry of the matrix $\boldsymbol{B}$.

Lemma 3.2. For any two zonal functions $\psi(t)$ and $\phi(t)$ of the form (10), we have the following relation:

$$
\int_{S^{n}} \psi(x \cdot z) \phi(z \cdot y) d S(z)=\frac{1}{\omega_{n}} \sum_{\ell=0}^{\infty} N(n, \ell) \widehat{\psi}(\ell) \widehat{\phi}(\ell) P_{\ell}(n+1 ; x \cdot y) .
$$

Proof. From the definition, $\psi(x \cdot z)$ and $\phi(y \cdot z)$ have the following expansions in terms of spherical harmonics:

$$
\begin{aligned}
& \psi(x \cdot z)=\sum_{\ell=0}^{\infty} \sum_{k=1}^{N(n, \ell)} \widehat{\psi}(\ell) Y_{\ell, k}(x) Y_{\ell, k}(z), \\
& \phi(y \cdot z)=\sum_{\ell=0}^{\infty} \sum_{k=1}^{N(n, \ell)} \widehat{\phi}(\ell) Y_{\ell, k}(y) Y_{\ell, k}(z) .
\end{aligned}
$$


Then, by using the orthogonality of spherical harmonics and the addition formula (3), we obtain

$$
\begin{aligned}
\int_{S^{n}} \psi(x \cdot z) \phi(z \cdot y) d S(z) & =\sum_{\ell=0}^{\infty} \sum_{k=1}^{N(n, \ell)} \widehat{\psi}(\ell) \widehat{\phi}(\ell) Y_{\ell, k}(x) Y_{\ell, k}(y) \\
& =\frac{1}{\omega_{n}} \sum_{\ell=0}^{\infty} N(n, \ell) \widehat{\psi}(\ell) \widehat{\phi}(\ell) P_{\ell}(n+1 ; x \cdot y) .
\end{aligned}
$$

If the supports of $\Phi_{i}$ and $\Phi_{j}$ do not overlap, then $B_{i, j}=0$. This follows from $a\left(\Phi_{i}, \Phi_{j}\right)=\left\langle-\Delta^{*} \Phi_{i}+\omega^{2} \Phi_{i}, \Phi_{j}\right\rangle$, together with the fact that the support of $\Delta^{*} \Phi_{i}$ is the same as that of $\Phi_{i}$. (This follows from the second-order differential operator form of the Laplace-Beltrami operator.) If the supports of $\Phi_{i}$ and $\Phi_{j}$ overlap, then, by applying Lemma 3.2 we have

$$
\begin{aligned}
B_{i, j} & =\int_{S^{n}}\left(-\Delta^{*}+\omega^{2}\right) \phi\left(x_{i} \cdot x\right) \phi\left(x_{j} \cdot x\right) d S(x) \\
& =\frac{1}{\omega_{n}} \sum_{\ell=0}^{\infty} N(n, \ell)\left(\lambda_{\ell}+\omega^{2}\right)[\widehat{\phi}(\ell)]^{2} P_{\ell}\left(n+1 ; x_{i} \cdot x_{j}\right) .
\end{aligned}
$$

\section{The abstract framework of ADditive Schwarz methods}

Additive Schwarz methods provide fast solutions to equation (20) by solving, at the same time, problems of smaller size. Let the space $V_{X}$ be decomposed as

$$
V_{X}=V_{0}+\cdots+V_{J}
$$

where $V_{j}, j=0, \ldots, J$, are subspaces of $V_{X}$, and let $P_{j}: V_{X} \rightarrow V_{j}, j=0, \ldots, J$, be projections defined by

$$
a\left(P_{j} v, w\right)=a(v, w) \quad \forall v \in V_{X}, \forall w \in V_{j} .
$$

If we define

$$
P:=P_{0}+\cdots+P_{J}
$$

then the additive Schwarz method for equation (20) consists of solving, by an iterative method, the equation

$$
P u_{X}=g,
$$

where the right-hand side is given by $g=\sum_{j=0}^{J} g_{j}$, with $g_{j} \in V_{j}$ being solutions of

$$
a\left(g_{j}, w\right)=\langle f, w\rangle \text {, for any } w \in V_{j} .
$$

The well-known equivalence of (20) and (26) was discussed explicitly in 25. In fact, if $u_{X}$ is a solution of (20), then from the definition of $P_{j}$ and $g_{j}$ we deduce

$$
a\left(P_{j} u_{X}, w\right)=a\left(u_{X}, w\right)=\langle f, w\rangle=a\left(g_{j}, w\right) \text { for any } w \in V_{j},
$$

i.e. $P_{j} u_{X}=g_{j}$. Hence $P u_{X}=g$. On the other hand, if $P: V_{X} \rightarrow V_{X}$ is invertible and $u_{X}$ is the solution of (26), then by using successively the symmetry of $P$ and 
(24) and (27), we obtain

$$
\begin{aligned}
a\left(u_{X}, v\right) & =a\left(P^{-1} g, v\right)=a\left(g, P^{-1} v\right) \\
& =\sum_{j=0}^{J} a\left(g_{j}, P^{-1} v\right)=\sum_{j=0}^{J} a\left(g_{j}, P_{j} P^{-1} v\right) \\
& =\sum_{j=0}^{J}\left\langle f, P_{j} P^{-1} v\right\rangle=\langle f, v\rangle \text { for any } v \in V_{X} .
\end{aligned}
$$

A practical method to solve (26) is the conjugate gradient method; the additive Schwarz method (see Section 6) can be viewed as a preconditioned conjugate gradient method.

Bounds for $\lambda_{\min }(P)$ and $\lambda_{\max }(P)$, the minimum and maximum eigenvalues of the additive Schwarz operator $P$, can be obtained by using the following lemma; see [17, 30].

Lemma 4.1. (i) Assume that there exists a constant $c_{1}>0$ such that, for any $u \in V_{X}$ satisfying $u=\sum_{j=0}^{J} u_{j}$ with $u_{j} \in V_{j}$ for $j=0, \ldots, J$ the following inequality

$$
a(u, u) \leq c_{1} \sum_{j=0}^{J} a\left(u_{j}, u_{j}\right)
$$

holds. Then

$$
\lambda_{\max }(P) \leq c_{1} .
$$

(ii) Assume that there exists a constant $c_{2}>0$ such that any $u \in V_{X}$ has a decomposition $u=\sum_{j=0}^{J} u_{j}, u_{j} \in V_{j}$, satisfying

$$
\sum_{j=0}^{J} a\left(u_{j}, u_{j}\right) \leq c_{2} a(u, u) .
$$

Then

$$
\lambda_{\min }(P) \geq c_{2}^{-1} .
$$

\section{Additive Schwarz method for elliptic PDEs on the Unit SPhere}

In this section we will define a subspace decomposition of the form (23), and in this way define the additive Schwarz operator for problem (20). We will then present the main theoretical result of the paper, namely an estimate for the condition number of the additive Schwarz operator.

5.1. Subspace decomposition. Let $\alpha$ be a fixed number satisfying $0<\alpha<\pi / 2$, and let $X_{0}:=\left\{p_{j}: j=1, \ldots, J\right\}$ be a subset of $X$ such that

$$
S^{n}=\bigcup_{j=1}^{J} C\left(p_{j}, \alpha\right) .
$$

Assume that the support of $\Phi(p, \cdot)$, which is a spherical cap centered at $p$, has radius $\gamma$. (In the case of SBF constructed from Wendland's radial basis functions, $\gamma=\pi / 3$.)

For $j=1, \ldots, J$, the subset $X_{j}$ is defined as

$$
X_{j}:=\left\{x_{k} \in X: \theta\left(x_{k}, p_{j}\right) \leq \alpha\right\} .
$$


The sets $X_{j}$ may have different numbers of elements and may overlap each other. Because of (28) it is clear that $X$ is decomposed into $J$ overlapping subsets $\left\{X_{j}\right.$ : $j=1, \ldots, J\}$ of discrete points such that

$$
X=\bigcup_{j=1}^{J} X_{j}
$$

We define $V_{j}=V_{X_{j}}, j=0, \ldots, J$; i.e., $V_{j}=$ span $\left\{\Phi_{k}: x_{k} \in X_{j}\right\}$, so that $V_{X}=V_{0}+\cdots+V_{J}$. The Schwarz operator $P$ is then defined by (24) and (25). Functions in $V_{j}$ have supports in $\Gamma_{j}$, where

$$
\Gamma_{j}:=C\left(p_{j}, \alpha+\gamma\right), \quad j=1, \ldots, J .
$$

We assume that:

Assumption 5.1. We can partition the index set $\{1, \ldots, J\}$ into $M$ (for $1 \leq M \leq$ $J)$ sets $J_{m}$, for $1 \leq m \leq M$ such that if $i, j \in J_{m}$ and $i \neq j$, then $\Gamma_{i} \cap \Gamma_{j}=\emptyset$.

The partitioning problem mentioned in Assumption 5.1 is related to the graph coloring problem [2]. We can define an undirected graph $G=(V, E)$ in which the set of vertices $V=\left\{\nu_{1}, \ldots, \nu_{J}\right\}$ is identified with the set of caps $\Gamma_{j}$, and $E$ is the set of edges, where if $\Gamma_{i} \cap \Gamma_{j} \neq \emptyset$, then there is an edge between $\nu_{i}$ and $\nu_{j}$. A partition satisfying Assumption 5.1 is equivalent to a coloring of the vertices of $G$ so that adjacent vertices have different colors. The minimal number of colors needed is called the chromatic number of $G$ and is denoted by $\delta(G)$. In general, it is difficult to determine the chromatic number of a graph. However, it is easy to see that

$$
\delta(G) \geq \omega(G),
$$

where $\omega(G)$ is the maximal order of a complete subgraph of $G$; that is, it is the maximal number of vertices all of which are mutually connected. In terms of the caps, every point on the sphere $S^{n}$ lies in at most $\omega(G)$ spherical caps $\Gamma_{j}$.

An upper bound of $\delta(G)$ is given in [2, Theorem 3, Chapter 5]: when $G$ is neither a complete graph nor an odd cycle, then $\delta(G) \leq \Delta(G)$, with $\Delta(G)$ being the maximal degree of $G$. In terms of our spherical caps, each cap $\Gamma_{j}$ intersects at most $\Delta(G)$ other caps.

Therefore, for a given set $X_{0}$ and parameters $\alpha, \gamma$, we can compute the lower bound $M_{1}:=\omega(G)$ and the upper bound $M_{2}:=\Delta(G)$ so that

$$
1 \leq M_{1} \leq M \leq M_{2} \leq J
$$

5.2. A bound for $\lambda_{\max }(P)$. We now state a lemma that will lead to an upper bound of the maximum eigenvalue $\lambda_{\max }(P)$.

Lemma 5.1. There exists a positive constant $c$ independent of the set $X$ such that for any $u \in V_{X}$ satisfying $u=\sum_{j=0}^{J} u_{j}$ with $u_{j} \in V_{j}$ for $j=0, \ldots, J$,

$$
a(u, u) \leq c M \sum_{j=0}^{J} a\left(u_{j}, u_{j}\right) .
$$

Proof. Using the inequality $|a+b|^{2} \leq 2\left(|a|^{2}+|b|^{2}\right)$, we have

$$
\|u\|_{H^{1}\left(S^{n}\right)}^{2} \leq 2\left(\left\|u_{0}\right\|_{H^{1}\left(S^{n}\right)}^{2}+\left\|\sum_{j=1}^{J} u_{j}\right\|_{H^{1}\left(S^{n}\right)}^{2}\right) .
$$


From the definition of the Sobolev norm (9),

$$
\left\|\sum_{j=1}^{J} u_{j}\right\|_{H^{1}\left(S^{n}\right)}^{2}=\left\|\sum_{j=1}^{J} \pi_{1}\left(\chi_{1} u_{j}\right)\right\|_{H^{1}\left(\mathbb{R}^{n}\right)}^{2}+\left\|\sum_{j=1}^{J} \pi_{2}\left(\chi_{2} u_{j}\right)\right\|_{H^{1}\left(\mathbb{R}^{n}\right)}^{2} .
$$

Now, from the fact that $u_{j} \in V_{j}$ together with Assumption 5.1 we can partition the index set $\{1, \ldots, J\}$ into $M$ sets of indices $J_{m}$ so that if $i, j \in J_{m}$, then supp $u_{i} \cap$ supp $u_{j}=\emptyset$. Then, in this proof only, let $g_{j}=\pi_{1}\left(\chi_{1} u_{j}\right)$. By using the CauchySchwarz inequality, we have

$$
\left\|\sum_{j=1}^{J} g_{j}\right\|_{H^{1}\left(\mathbb{R}^{n}\right)}^{2}=\left\|\sum_{m=1}^{M} \sum_{j \in J_{m}} g_{j}\right\|_{H^{1}\left(\mathbb{R}^{n}\right)}^{2} \leq M \sum_{m=1}^{M}\left\|\sum_{j \in J_{m}} g_{j}\right\|_{H^{1}\left(\mathbb{R}^{n}\right)}^{2} .
$$

Since the supports of $g_{i}$ and $g_{j}$ are disjoint for $i, j \in J_{m}, i \neq j$,

$$
\left\|\sum_{j \in J_{m}} g_{j}\right\|_{H^{1}\left(\mathbb{R}^{n}\right)}^{2}=\sum_{j \in J_{m}}\left\|g_{j}\right\|_{H^{1}\left(\mathbb{R}^{n}\right)}^{2} .
$$

Thus,

$$
\left\|\sum_{j=1}^{J} g_{j}\right\|_{H^{1}\left(\mathbb{R}^{n}\right)}^{2} \leq M \sum_{m=1}^{M} \sum_{j \in J_{m}}\left\|g_{j}\right\|_{H^{1}\left(\mathbb{R}^{n}\right)}^{2}=M \sum_{j=1}^{J}\left\|g_{j}\right\|_{H^{1}\left(\mathbb{R}^{n}\right)}^{2} .
$$

Hence, by using similar arguments for $\pi_{2}\left(\chi_{2} u_{j}\right)$, we conclude

$$
\begin{aligned}
\left\|\sum_{j=1}^{J} u_{j}\right\|_{H^{1}\left(S^{n}\right)}^{2} & \leq M\left(\sum_{j=1}^{J}\left\|\pi_{1}\left(\chi_{1} u_{j}\right)\right\|_{H^{1}\left(\mathbb{R}^{n}\right)}^{2}+\sum_{j=1}^{J}\left\|\pi_{2}\left(\chi_{2} u_{j}\right)\right\|_{H^{1}\left(\mathbb{R}^{n}\right)}^{2}\right) \\
& =M \sum_{j=1}^{J}\left\|u_{j}\right\|_{H^{1}\left(S^{n}\right)}^{2} .
\end{aligned}
$$

Therefore,

$$
\|u\|_{H^{1}\left(S^{n}\right)}^{2} \leq c M \sum_{j=0}^{J}\left\|u_{j}\right\|_{H^{1}\left(S^{n}\right)}^{2} .
$$

Using the fact that $a(u, u) \sim\|u\|_{H^{1}\left(S^{n}\right)}^{2}$ we obtain the result.

From this lemma and Lemma 4.1 it follows that

$$
\lambda_{\max }(P) \leq c M
$$

where $c$ is a constant independent of $M, J$ and the set $X$.

5.3. A bound for $\lambda_{\min }(P)$. We now find a lower bound for the minimum eigenvalue of $P$. In the finite element and boundary element literature, a lower bound is usually obtained by using the interpolation operator and a partition of unity; see, e.g., 23, 26. More precisely, in the FEM and BEM cases a decomposition of a function $u \in V_{X}$ satisfying the condition of Lemma 4.1 (ii) takes the following form:

$$
u_{0}=P_{0} u, \quad u_{j}=\Pi_{X}\left(\varphi_{j}\left(u-u_{0}\right)\right), j=1, \ldots, J
$$


where $P_{0}$ is the $H^{1}$-projection onto $V_{0}, \Pi_{X}$ is the interpolation operator at the data points, and $\left\{\varphi_{j}: j=1, \ldots, J\right\}$ is a partition of unity satisfying $\operatorname{supp} \varphi_{j} \subset \Gamma_{j}$. This approach cannot be used in the present case because with radial basis functions the support of $\Pi_{X}(v)$ may be the whole sphere even though the support of $v$ is in $\Gamma_{j}$. The method of alternating projections [4, 18, will be used instead. Before introducing this method we need the following definition.

Definition 5.1. Let $\mathcal{V}$ be a Hilbert space with inner product and norm denoted by $\langle\cdot, \cdot\rangle$ and $\|\cdot\|$, respectively. Assume that $\mathcal{U}_{1}$ and $\mathcal{U}_{2}$ are two closed subspaces of $\mathcal{V}$. The angle $\alpha$ between $\mathcal{U}_{1}$ and $\mathcal{U}_{2}$ is the angle in $[0, \pi / 2]$ whose cosine is given by

$$
\cos \alpha=\sup \left\{\langle v, w\rangle: v \in \mathcal{U}_{1} \cap \mathcal{U}^{\perp}, \quad w \in \mathcal{U}_{2} \cap \mathcal{U}^{\perp}, \quad\|v\| \leq 1,\|w\| \leq 1\right\},
$$

where $\mathcal{U}=\mathcal{U}_{1} \cap \mathcal{U}_{2}$, and $\mathcal{U}^{\perp}$ is its orthogonal complement, namely,

$$
\mathcal{U}^{\perp}:=\{f \in \mathcal{V}:\langle f, v\rangle=0 \quad \forall v \in \mathcal{U}\} .
$$

The following result is standard; the proof is included for completeness.

Lemma 5.2. If $\mathcal{U}_{1}$ and $\mathcal{U}_{2}$ are two closed subspaces of a Hilbert space $\mathcal{V}$, then

$$
\left(\mathcal{U}_{1}+\mathcal{U}_{2}\right)^{\perp}=\mathcal{U}_{1}^{\perp} \cap \mathcal{U}_{2}^{\perp} .
$$

Proof. It follows from the definition of the orthogonal complement that

$$
\begin{aligned}
\left(\mathcal{U}_{1}+\mathcal{U}_{2}\right)^{\perp} & =\left\{f \in \mathcal{V}:\langle f, v\rangle=0 \quad \forall v \in \mathcal{U}_{1}+\mathcal{U}_{2}\right\} \\
& =\left\{f \in \mathcal{V}:\left\langle f, v_{1}\right\rangle=0=\left\langle f, v_{2}\right\rangle \quad \forall v_{1} \in \mathcal{U}_{1} \text { and } v_{2} \in \mathcal{U}_{2}\right\} \\
& =\mathcal{U}_{1}^{\perp} \cap \mathcal{U}_{2}^{\perp} .
\end{aligned}
$$

The following theorem is crucial in our estimate of the minimum eigenvalue of $P$.

Theorem 5.1 (21, Theorem 2.2]). Let $\mathcal{V}_{1}, \ldots, \mathcal{V}_{J}$ be closed subspaces of a Hilbert space $\mathcal{V}$, and $\mathcal{W}_{i}:=\bigcap_{j=i}^{J} \mathcal{V}_{j}, i=1, \ldots, J$. If $Q_{i}: \mathcal{V} \rightarrow \mathcal{V}_{i}$ is the orthogonal projection onto $\mathcal{V}_{i}, i=1, \ldots, J$, and $Q: \mathcal{V} \rightarrow \mathcal{W}_{1}$ is the orthogonal projection onto $\mathcal{W}_{1}$, then

$$
\left\|\widetilde{Q}^{l} f-Q f\right\| \leq c^{l}\|f-Q f\|, \quad \forall f \in \mathcal{V}, \quad l=1,2, \ldots,
$$

where $\widetilde{Q}:=Q_{J} \cdots Q_{1}$ and

$$
c^{2}=1-\prod_{i=1}^{J-1} \sin ^{2} \alpha_{i}
$$

with $\alpha_{i}$ being the angle between $\mathcal{V}_{i}$ and $\mathcal{W}_{i+1}$.

We shall apply Theorem 5.1 with $\mathcal{V}$ being $V_{X}$, which is equipped with the inner product $a(\cdot, \cdot)$ and induced norm $\|\cdot\|_{a}$, and $\mathcal{V}_{j}$ being $V_{j}^{\perp}, j=1, \ldots, J$. If $T$ is a linear operator on $V_{X}$, we denote by $\|T\|_{a}$ the norm of $T$ defined by $\|\cdot\|_{a}$, i.e.,

$$
\|T\|_{a}=\sup _{\substack{v \in V_{X} \\\|v\|_{a} \leq 1}}\|T v\|_{a}
$$

Proposition 5.1. Let $\widetilde{Q}:=Q_{J} \cdots Q_{1}$, where $Q_{i}$ is the orthogonal projection from $V_{X}$ onto $V_{i}^{\perp}$, and let $W_{i}:=\bigcap_{j=i}^{J} V_{j}^{\perp}, i=1, \ldots, J$. Then

$$
\|\widetilde{Q}\|_{a} \leq\left(1-\prod_{i=1}^{J-1} \sin ^{2} \alpha_{i}\right)^{1 / 2}<1,
$$

where $\alpha_{i}$ is the angle between $V_{i}^{\perp}$ and $W_{i+1}$. 
Proof. It follows from Lemma 5.2 that

$$
W_{1}=\bigcap_{j=1}^{J} V_{j}^{\perp}=\left(V_{1}+\cdots+V_{J}\right)^{\perp}=V_{X}^{\perp}=\{0\},
$$

which implies that the orthogonal projection $Q$ from $V_{X}$ onto $W_{1}$ is identically zero. Theorem 5.1 with $l=1$ then yields

$$
\|\widetilde{Q}\|_{a} \leq\left(1-\prod_{i=1}^{J-1} \sin ^{2} \alpha_{i}\right)^{1 / 2} .
$$

It remains to show that $\alpha_{i} \neq 0$ for all $i=1, \ldots, J-1$. Suppose that $\alpha_{i}=0$ for some $i \in\{1, \ldots, J-1\}$. Then noting that

$$
\left(V_{i}^{\perp} \cap W_{i+1}\right)^{\perp}=W_{i}^{\perp},
$$

we obtain from Definition 5.1 ,

$$
\sup \left\{a(v, w): v \in V_{i}^{\perp} \cap W_{i}^{\perp}, w \in W_{i+1} \cap W_{i}^{\perp},\|v\|_{a} \leq 1,\|w\|_{a} \leq 1\right\}=1 .
$$

The spaces being finite dimensional, by compactness there exist $v \in V_{i}^{\perp} \cap W_{i}^{\perp}$ and $w \in W_{i+1} \cap W_{i}^{\perp}$ satisfying

$$
\|v\|_{a}=\|w\|_{a}=1 \quad \text { and } \quad a(v, w)=1 .
$$

It follows from the Cauchy-Schwarz inequality that $v=w$. Thus $v \in V_{i}^{\perp} \cap W_{i+1}=$ $W_{i}$. On the other hand, $v \in W_{i}^{\perp}$, which implies $v=0$. This contradicts $a(v, v)=1$, proving the proposition.

Lemma 5.3. For any $u \in V_{X}$ there exist $u_{j} \in V_{j}, j=0, \ldots, J$, satisfying $u=$ $\sum_{j=0}^{J} u_{j}$ and

$$
\sum_{j=0}^{J} a\left(u_{j}, u_{j}\right) \leq\left(1+\frac{J}{\left(1-\|\widetilde{Q}\|_{a}\right)^{2}}\right) a(u, u),
$$

where $\widetilde{Q}$ is defined in Proposition 5.1 .

Proof. It follows from Proposition 5.1 that $I-\widetilde{Q}$ is invertible and satisfies

$$
\left\|(I-\widetilde{Q})^{-1}\right\|_{a} \leq \frac{1}{1-\|\widetilde{Q}\|_{a}},
$$

where $I$ is the identity operator on $V_{X}$. We define, for any $u \in V_{X}$,

$$
\begin{aligned}
& u_{0}=P_{0} u, \quad v=u-u_{0}, \\
& u_{1}=P_{1}(I-\widetilde{Q})^{-1} v, \\
& u_{j}=P_{j} Q_{j-1} \cdots Q_{1}(I-\widetilde{Q})^{-1} v, j=2, \ldots, J,
\end{aligned}
$$

where $P_{i}:=I-Q_{i}$ is the orthogonal projection from $V_{X}$ onto $V_{i}, i=0, \ldots, J$. It is easy to check that $\sum_{j=1}^{J} u_{j}$ (being a telescoping sum) equals $v$, and therefore $\sum_{i=0}^{J} u_{i}=u$. The crude estimate

$$
\left\|u_{j}\right\|_{a} \leq\left\|(I-\widetilde{Q})^{-1}\right\|_{a}\|v\|_{a} \leq\left\|(I-\widetilde{Q})^{-1}\right\|_{a}\|u\|_{a}, \quad j=1, \ldots, J,
$$

yields

$$
a\left(u_{j}, u_{j}\right) \leq \frac{1}{\left(1-\|\widetilde{Q}\|_{a}\right)^{2}} a(u, u), \quad j=1, \ldots, J
$$


resulting in

$$
\begin{aligned}
\sum_{j=0}^{J} a\left(u_{j}, u_{j}\right) & =a\left(u_{0}, u_{0}\right)+\sum_{j=1}^{J} a\left(u_{j}, u_{j}\right) \\
& \leq\left(1+\frac{J}{\left(1-\|\widetilde{Q}\|_{a}\right)^{2}}\right) a(u, u)
\end{aligned}
$$

proving the lemma.

The above lemma and Lemma 4.1 yield the following estimate for the minimum eigenvalue of $P$ :

$$
\lambda_{\min }(P) \geq\left(1+\frac{J}{\left(1-\|\widetilde{Q}\|_{a}\right)^{2}}\right)^{-1} .
$$

This estimate is by no means sharp; in fact, the right-hand side is not an optimal lower bound for $\lambda_{\min }(P)$, as can be seen in Table 1, because $\|\widetilde{Q}\|_{a}$ may be very close to 1 . In that table we present $\lambda_{\min }^{-1}(P), 1+\sum_{j=1}^{J} C_{j}^{2}$ and $1+\frac{J}{\left(1-\|\tilde{Q}\|_{a}\right)^{2}}$, where for the middle term we explicitly compute $C_{j}$ as the norm of the operator defining $u_{j}$, namely,

$$
C_{1}=\left\|P_{1}(I-\widetilde{Q})^{-1}\right\|_{a} \quad \text { and } \quad C_{j}=\left\|P_{j} Q_{j-1} \cdots Q_{1}(I-\widetilde{Q})^{-1}\right\|_{a}, j=2, \ldots, J .
$$

It is clear from Table 1 that $\left(1+\sum_{j=1}^{J} C_{j}^{2}\right)^{-1}$ is a better approximant to $\lambda_{\min }(P)$. Our experiments show that the projection $P_{j}$ in the definition of $u_{j}$ plays a key role in reducing the norm of $(I-\widetilde{Q})^{-1}$, but we cannot account for this fact.

In Table 1 the norms of the operators were computed by using their matrix representations. E.g., we computed $\|\widetilde{Q}\|_{a}$ as follows. Recalling the definition of the positive definite matrix $\boldsymbol{B}$ (see (21)) and using the Cholesky factorization $\boldsymbol{B}=$ $\boldsymbol{L}^{T} \boldsymbol{L}$, we obtain for any $u=\sum_{i=1}^{N} c_{i} \Phi_{i} \in V_{X}$,

$$
\|u\|_{a}^{2}=\boldsymbol{c}^{T} \boldsymbol{B} \boldsymbol{c}=\|\boldsymbol{L} \boldsymbol{c}\|_{\ell_{2}}^{2},
$$

where $\boldsymbol{c}=\left(c_{1}, \ldots, c_{N}\right)^{T}$. On the other hand, by writing

$$
\widetilde{Q} \Phi_{i}=\sum_{k=1}^{N} d_{i, k} \Phi_{k},
$$

one can easily see that

$$
\|\widetilde{Q} u\|_{a}^{2}=\boldsymbol{c}^{T} \widetilde{\boldsymbol{Q}}^{T} \boldsymbol{B} \widetilde{\boldsymbol{Q}} \boldsymbol{c}=\boldsymbol{c}^{T} \widetilde{\boldsymbol{Q}}^{T} \boldsymbol{L}^{T} \boldsymbol{L} \widetilde{\boldsymbol{Q}} \boldsymbol{c}=\|\boldsymbol{L} \widetilde{\boldsymbol{Q}} \boldsymbol{c}\|_{\ell_{2}}^{2},
$$

where $\widetilde{\boldsymbol{Q}}$ is the matrix representation of $\widetilde{Q}$ with the $i$ th column being $\left(d_{i, 1}, \ldots\right.$, $\left.d_{i, N}\right)^{T}$. Therefore,

$$
\|\widetilde{Q}\|_{a}=\sup _{u \in V_{X}} \frac{\|\widetilde{Q} u\|_{a}}{\|u\|_{a}}=\sup _{\boldsymbol{c} \in \mathbb{R}^{N}} \frac{\|\boldsymbol{L} \widetilde{\boldsymbol{Q}} \boldsymbol{c}\|_{\ell_{2}}}{\|\boldsymbol{L} \boldsymbol{c}\|_{\ell_{2}}}=\sup _{\boldsymbol{c} \in \mathbb{R}^{N}} \frac{\left\|\boldsymbol{L} \widetilde{\boldsymbol{Q}} \boldsymbol{L}^{-1} \boldsymbol{c}\right\|_{\ell_{2}}}{\|\boldsymbol{c}\|_{\ell_{2}}}=\left\|\boldsymbol{L} \widetilde{\boldsymbol{Q}} \boldsymbol{L}^{-1}\right\|_{\ell_{2}} .
$$


TABLE 1. Upper bounds for $\lambda_{\min }^{-1}(P)$

\begin{tabular}{|c|c|c|c|c|c|r|r|}
\hline$N$ & $q_{X}$ & $\cos \alpha$ & $\cos \beta$ & $J$ & $\lambda_{\min }^{-1}(P)$ & $1+\sum_{j=1}^{J} C_{j}^{2}$ & $1+J /\left(1-\|\widetilde{Q}\|_{a}\right)^{2}$ \\
\hline 1344 & $\pi / 80$ & 0.90 & -0.90 & 44 & 120.41 & 1612.23 & 98914208.40 \\
& & 0.80 & -0.93 & 23 & 285.80 & 461.67 & 4003258.71 \\
& & 0.70 & -0.86 & 17 & 17.44 & 135.84 & 625605.77 \\
& & 0.60 & -0.87 & 13 & 13.03 & 33.11 & 7481.56 \\
& & 0.50 & -0.83 & 10 & 3.58 & 12.43 & 219.18 \\
\hline 2133 & $\pi / 100$ & 0.90 & -0.85 & 44 & 34.97 & 391.90 & 1684693.43 \\
& & 0.80 & -0.89 & 24 & 20.16 & 69.22 & 57266.95 \\
& & 0.70 & -0.89 & 17 & 6.56 & 24.09 & 712.68 \\
& & 0.60 & -0.85 & 13 & 8.18 & 26.44 & 4745.12 \\
& & 0.50 & -0.83 & 10 & 4.48 & 13.88 & 266.90 \\
\hline 3458 & $\pi / 140$ & 0.90 & -0.88 & 46 & 4.92 & 68.75 & 3662.59 \\
& & 0.80 & -0.80 & 22 & 5.31 & 41.58 & 2649.72 \\
& & 0.70 & -0.81 & 16 & 1.82 & 17.77 & 101.96 \\
& & 0.60 & -0.85 & 13 & 1.02 & 13.31 & 47.03 \\
& & 0.50 & -0.83 & 10 & 1.70 & 11.67 & 58.05 \\
\hline 4108 & $\pi / 160$ & 0.90 & -0.88 & 46 & 2.17 & 66.21 & 4023.85 \\
& & 0.80 & -0.81 & 24 & 6.41 & 36.54 & 1043.01 \\
& & 0.70 & -0.87 & 17 & 4.57 & 22.21 & 755.70 \\
& & 0.60 & -0.86 & 14 & 1.26 & 15.31 & 86.07 \\
& & 0.50 & -0.80 & 10 & 4.13 & 16.85 & 1388.24 \\
\hline 7663 & $\pi / 200$ & 0.80 & -0.89 & 23 & 1.37 & 27.47 & 154.84 \\
& & 0.70 & -0.88 & 17 & 1.68 & 19.32 & 130.77 \\
& & 0.60 & -0.81 & 11 & 5.54 & 17.35 & 1709.86 \\
& & 0.50 & -0.84 & 10 & 3.27 & 12.78 & \\
\hline
\end{tabular}

5.4. Main result. Estimates (33) and (34) yield an upper bound for the condition number of the Schwarz operator.

Theorem 5.2. The condition number of the additive Schwarz operator $P$ is bounded by

$$
\kappa(P) \leq c M\left(1+\frac{J}{\left(1-\|\widetilde{Q}\|_{a}\right)^{2}}\right)
$$

where $c$ is a constant independent of $M, J$ and the set $X$, and $\widetilde{Q}$ is defined as in Proposition 5.1.

\section{An Overlapping AdDitive Schwarz ALGOrithm}

As has been pointed out in [1, the essential ingredients for a domain decomposition algorithm are:

(i) A method for subdividing the physical space.

(ii) An efficient and scale independent method for solving small subproblems. The solutions to the small problems will be used to precondition the large problem. 


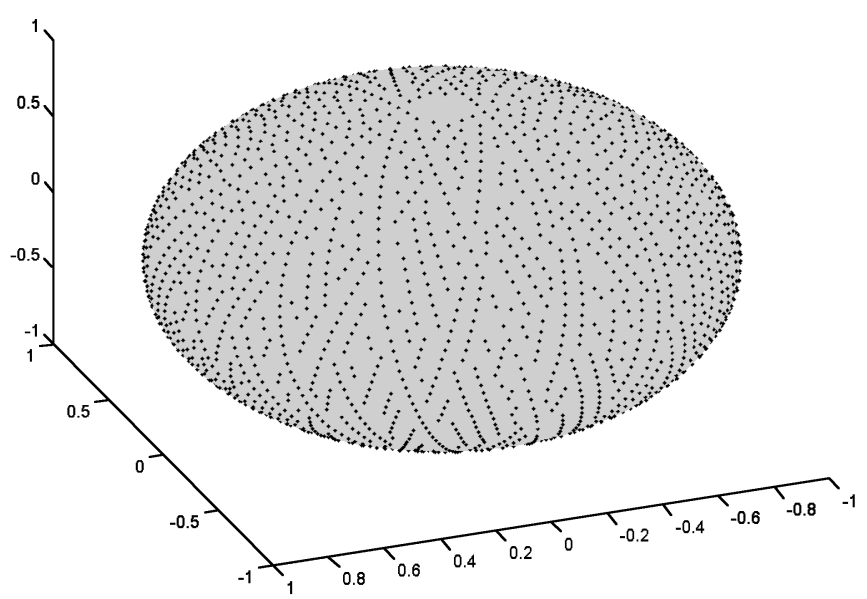

FigURE 1. Global scattered MAGSAT satellite data

(iii) A fast method for computing the action of the large matrices that occur at various scales.

(iv) An outer iteration.

Suppose we number the scattered data following the satellite track as $\{1, \ldots, N\}$. Let $\alpha$ and $\beta$ be parameters satisfying $0<\alpha<\pi / 3$ and $\alpha \leq \beta \leq \pi$. The algorithm to partition $X$ can be described as follows.

(1) The first center is $p_{1}=x_{1}$.

(2) Assume that the centers $p_{1}, \ldots, p_{l}$ have been chosen.

(3) If $X=\bigcup_{k=1}^{l} X_{k}$ (where $X_{k}$ is defined by (29)), then stop the algorithm and let $J=l$. Otherwise, choose the new center $p_{l+1}$ as a point in $X$ satisfying

$$
\theta\left(p_{l+1}, p_{l}\right) \geq \beta \quad \text { and } \theta\left(p_{l+1}, p_{k}\right) \geq \alpha, \quad k=1, \ldots, l-1 .
$$

(4) Let $l=l+1$ and repeat step (3).

Now $X_{0}$ is defined by $X_{0}:=\left\{p_{1}, \ldots, p_{J}\right\}$. The parameter $\beta$ is included so that the condition $X=\bigcup_{k=1}^{l} X_{k}$ in step (3) is quickly satisfied. In our experiments, for a given value of $\alpha$ we chose an appropriate value of $\beta$ by starting with the value $\beta=\pi$, and decreased its value until the above-mentioned condition holds.

For $j=0, \ldots, J$, let $I_{j}$ be a subset of the index set of $\{1, \ldots, N\}$ such that

$$
m \in I_{j} \leftrightarrow x_{m} \in X_{j} .
$$

The cardinality of the set $I_{j}$ is denoted by $s_{j}$ and the $m$-th element of the set $I_{j}$ is denoted by $I_{j}(m)$. For a given vector $\mathbf{v}=\left(v_{1}, \ldots, v_{N}\right)^{T}$, the restriction of $\mathbf{v}$ on $X_{j}$ is the vector $\mathbf{u}=\left(u_{1}, u_{2}, \ldots, u_{s_{j}}\right)^{T}$ defined as follows:

$$
u_{m}:=v_{I_{j}(m)}, \quad m=1, \ldots, s_{j},
$$

and we write $\mathbf{u}=R_{j}(\mathbf{v})$; thus the restriction operation to $X_{j}$ is denoted by $R_{j}$. Conversely, for a vector $\mathbf{u}=\left(u_{1}, \ldots, u_{s_{j}}\right)^{T}$, we extend $\mathbf{u}$ to $\mathbf{v}=\left(v_{1}, \ldots, v_{N}\right)^{T}$ as

$$
v_{k}:= \begin{cases}u_{m} & \text { if } k=I_{j}(m) \text { for } 1 \leq m \leq s_{j}, \\ 0 & \text { otherwise, }\end{cases}
$$

and write $\mathbf{v}=E_{j}(\mathbf{u})$, where $E_{j}$ denotes the extension operation. 


\section{A pseudocode.}

INPUT

Input the scattered set $X$ on the sphere, the right-hand side $f$, and the desired accuracy $\epsilon$.

SETUP

(1) Partition the scattered set $X$ into $J$ overlapping subsets $X_{j}$ for $j=1, \ldots, J$ and construct the global coarse set $X_{0}$.

(2) Set the initial residual vector $\mathbf{r}=\mathbf{f}$ (see (21)).

(3) Set the initial pseudoresidual vector $\mathbf{p}=\mathbf{0}$.

(4) Set the initial solution vector $\mathbf{s}=\mathbf{0}$.

(5) Set the iteration counter iter $=0$.

ITERATIVE SOLUTION

(1) while $\|\mathbf{r}\|>\epsilon\|\mathbf{f}\|$

(2) Set $\mathbf{p}=\mathbf{0}$.

(3) for $j=1$ to $J$

(4) Construct the local matrix $C$ with entries $C_{m, n}=B_{I_{j}(m), I_{j}(n)}$.

(5) Set the restriction residual vector $\mathbf{z}=R_{j}(\mathbf{r})$.

(6) Solve the linear system $C \mathbf{y}=\mathbf{z}$.

(7) Update the pseudoresidual vector $\mathbf{p}=\mathbf{p}+E_{j}(\mathbf{y})$.

(8) end for

(9) Construct the coarse global matrix $G$ with entries

$$
G_{m, n}=B_{I_{0}(m), I_{0}(n)} .
$$

(10) Set $\mathbf{z}_{g}=R_{0}(\mathbf{r})$.

(11) Solve the linear system $G \mathbf{y}_{g}=\mathbf{z}_{g}$.

(12) Update the pseudoresidual vector $\mathbf{p}=\mathbf{p}+E_{0}\left(\mathbf{y}_{g}\right)$.

(13) If iter $>0$, then set $\zeta_{0}=\zeta_{1}$.

(14) Set $\zeta_{1}=\mathbf{p} \cdot \mathbf{r}$.

(15) Update the counter, iter $=$ iter +1 .

(16) If iter $=1$, then define $\overline{\mathbf{p}}=\mathbf{p}$ else $\overline{\mathbf{p}}=\mathbf{p}+\left(\zeta_{1} / \zeta_{0}\right) \overline{\mathbf{p}}$.

(17) Update the residual vector

$$
\mathbf{r}=\mathbf{r}-\left(\frac{\mathbf{r} \cdot \mathbf{p}}{\overline{\mathbf{p}} \cdot B \overline{\mathbf{p}}}\right) B \overline{\mathbf{p}}
$$

Update the solution vector

$$
\mathbf{s}=\mathbf{s}+\left(\frac{\mathbf{r} \cdot \mathbf{p}}{\overline{\mathbf{p}} \cdot B \overline{\mathbf{p}}}\right) \overline{\mathbf{p}}
$$

(19) end while

\section{Numerical Results}

In this section, we present numerical experiments on $S^{2}$ based on globally scattered position data extracted from a very large data set collected by NASA's satellite MAGSAT. The scattered data sets $X$ are extracted so that the separation radius $q_{X}$ satisfies $q_{X} \geq q$, where $0<q<\pi / 3$ is a given number. The code is written in FORTRAN 90 and was run on computers equipped with dual Opteron $2.0 \mathrm{GHz}$ CPU and 4GB RAM. 


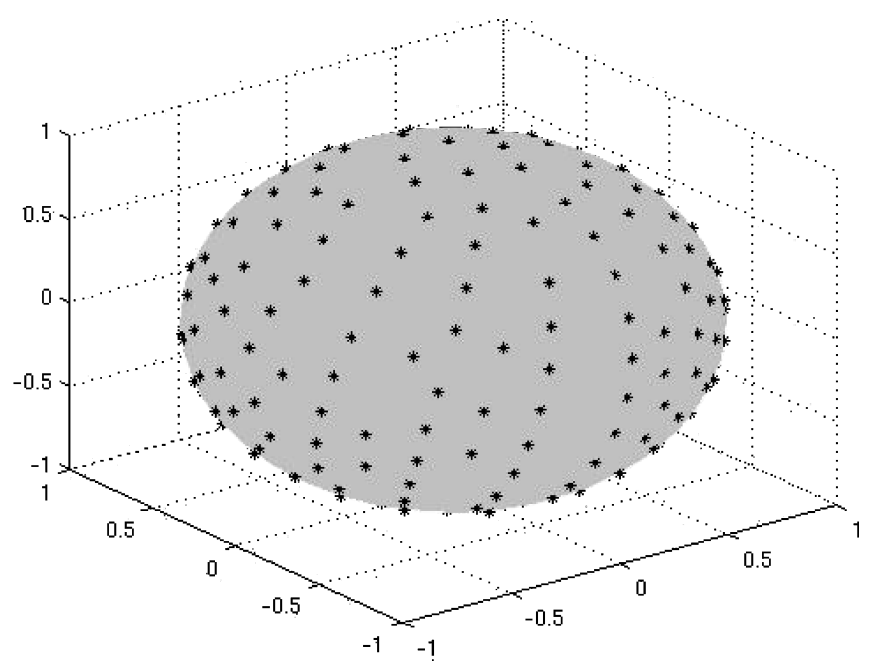

Figure 2 . The set $X_{0}$ with $N=10443, \cos \alpha=0.98$, and $\cos \beta=-0.70$

We wish to solve

$$
\left(-\Delta^{*}+I\right) u(x)=f(x), \quad x \in S^{2},
$$

with $I$ being the identity operator. The function $f$ is defined to be

$$
f(x)=f\left(x_{3}\right)=\left(-\Delta^{*}+I\right) \rho_{3,2}\left(\sqrt{2-2 x_{3}}\right), \quad x_{3} \in[-1,1],
$$

with $\rho_{3,2}(r)=(1-r)_{+}^{6}\left(35 r^{2}+18 r+3\right)$ so that the exact solution is

$$
u=\rho_{3,2}\left(\sqrt{2-2 x_{3}}\right) .
$$

The radial basis function $\Psi_{3,2}(x)=\rho_{3,2}(\|x\|)$ is in $C^{4}\left(\mathbb{R}^{3}\right)$ (see Section 2.3).

In the first set of numerical experiments, the spherical basis functions are derived from Wendland's radial basis function $\Psi_{3,3}(x)=\rho_{3,3}(\|x\|)$, which is in $C^{6}\left(\mathbb{R}^{3}\right)$ (see Section 2.3),

$$
\phi(x \cdot y)=\rho_{3,3}(\sqrt{2-2 x \cdot y}), \quad \rho_{3,3}(r)=(1-r)_{+}^{8}\left(32 r^{3}+25 r^{2}+8 r+1\right) .
$$

Recalling (22), and noting that with $n=2$ we have $N(n, \ell)=2 \ell+1$ and $\lambda_{\ell}=$ $\ell(\ell+1)$, each entry of the matrix $\boldsymbol{B}$ is given by

$$
\begin{aligned}
B_{i, j} & =\int_{S^{2}}\left(-\Delta^{*}+\omega^{2}\right) \phi\left(x_{i} \cdot x\right) \phi\left(x_{j} \cdot x\right) d S(x) \\
& =\frac{1}{4 \pi} \sum_{\ell=0}^{\infty}(2 \ell+1)\left(\lambda_{\ell}+\omega^{2}\right)[\widehat{\phi}(\ell)]^{2} P_{\ell}\left(3 ; x_{i} \cdot x_{j}\right) .
\end{aligned}
$$

In our experiments, $B_{i, j}$ is approximated by the partial sum of the first 3000 terms. The Legendre coefficients of the univariate function $\widehat{\phi}(\ell)$ were computed by approximating (11) by an appropriate Gaussian quadrature over the interval $[-1,1]$.

We then test the overlapping method as the preconditioner for the conjugate gradient method with different values of $\cos \alpha$, and hence different values of $J$ and $M$. The stopping criterion for the iteration is

$$
\frac{\left\|\boldsymbol{B c}^{(m)}-\mathbf{f}\right\|_{\ell^{2}}}{\|\mathbf{f}\|_{\ell^{2}}} \leq 10^{-6},
$$


where $\boldsymbol{B}$ and $\mathbf{f}$ are the stiffness matrix and the right-hand side vector as in (21). In the following tables, $\kappa(P)$ is the condition number, the CPU time is measured in seconds, and iter is the number of iterations. The results for the first experiment are shown in Table 2, Here $M_{1}$ and $M_{2}$ are the lower and upper bounds on $M$ from (30).

TABLE 2. Condition numbers of the Schwarz operator using $\rho_{3,3}(r)$ as RBF

\begin{tabular}{|c|c|c|c|c|c|c|c|c|c|c|c|}
\hline$N$ & $q_{X}$ & $\cos \alpha$ & $\cos \beta$ & $M_{1}$ & $M_{2}$ & $J$ & $\lambda_{\min }$ & $\lambda_{\max }$ & $\kappa(P)$ & $\mathrm{CPU}$ & Iter. \\
\hline 10443 & $\frac{\pi}{40}$ & 0.99 & -0.52 & 140 & 367 & 420 & 0.009 & 44.37 & 4693.57 & 116.2 & 171 \\
& & 0.98 & -0.70 & 86 & 205 & 223 & 0.053 & 29.48 & 559.95 & 68.4 & 93 \\
& & 0.97 & -0.64 & 56 & 131 & 138 & 0.034 & 21.78 & 645.84 & 78.2 & 90 \\
& & 0.95 & -0.63 & 43 & 91 & 94 & 0.122 & 17.62 & 145.04 & 70.0 & 51 \\
& & 0.85 & -0.93 & 19 & 30 & 31 & 0.590 & 10.19 & 17.26 & 141.2 & 21 \\
& & 0.60 & -0.86 & 13 & 14 & 15 & 1.076 & 9.02 & 8.38 & 865.0 & 16 \\
\hline 13775 & $\frac{\pi}{280}$ & 0.99 & -0.40 & 141 & 374 & 429 & 0.031 & 44.39 & 1420.38 & 153.9 & 132 \\
& & 0.98 & -0.51 & 85 & 206 & 226 & 0.092 & 29.20 & 316.61 & 103.4 & 76 \\
& & 0.97 & -0.57 & 56 & 132 & 140 & 0.069 & 21.57 & 310.99 & 108.2 & 66 \\
& & 0.95 & -0.62 & 44 & 93 & 96 & 0.210 & 18.23 & 86.94 & 124.9 & 45 \\
& & 0.85 & -0.89 & 21 & 33 & 34 & 0.636 & 10.73 & 16.86 & 350.7 & 22 \\
& & 0.60 & -0.77 & 11 & 12 & 13 & 0.909 & 8.38 & 9.22 & 1761.7 & 17 \\
\hline 17078 & $\frac{\pi}{320}$ & 0.99 & -0.44 & 148 & 387 & 442 & 0.037 & 46.50 & 1272.70 & 222.6 & 128 \\
& & 0.98 & -0.71 & 85 & 207 & 227 & 0.074 & 30.58 & 410.70 & 185.3 & 88 \\
& & 0.97 & -0.52 & 59 & 137 & 146 & 0.070 & 23.29 & 333.57 & 196.2 & 72 \\
& & 0.95 & -0.81 & 44 & 93 & 96 & 0.161 & 17.72 & 109.74 & 233.6 & 48 \\
& & 0.85 & -0.92 & 20 & 31 & 32 & 0.477 & 10.47 & 21.97 & 702.0 & 24 \\
& & 0.60 & -0.79 & 11 & 12 & 13 & 0.623 & 8.42 & 13.51 & 3924.0 & 20 \\
\hline
\end{tabular}

In the second set of experiments, the spherical basis function is derived from a less smooth radial basis function $\Psi_{3,2} \in C^{4}\left(\mathbb{R}^{3}\right)$,

$$
\phi(x \cdot y)=\rho_{3,2}(\sqrt{2-2 x \cdot y}), \quad \rho_{3,2}(r)=(1-r)_{+}^{6}\left(35 r^{2}+18 r+3\right) .
$$

The results are shown in Table 3 .

The numbers in both Tables 2 and 3 suggest that when $\cos \alpha$ decreases (meaning that $\alpha$ increases), $M_{1}, M_{2}$ and $\kappa(P)$ decrease, but the CPU time decreases then increases. We note that a larger value of $\alpha$ results in a larger size of the overlap and a smaller value of $J$ (the number of subproblems to be solved), which in turn implies larger sizes of the subproblems. As in the case of finite element methods, this results in a smaller condition number $\kappa(P)$ because the preconditioner is closer to the inverse of the stiffness matrix. However, for an optimal value of $\alpha$ in term of CPU time, one has to balance between the number of subproblems and their sizes. Our experiments show that any value of $\alpha$ so that $\cos \alpha \leq 0.60$ is not recommended. Optimal CPU times seem to occur when $\cos \alpha=0.98$. The numbers in Tables 2 and 3 also suggest that the smoothness of the kernel does not affect the algorithm.

Table 4 shows the CPU time and condition number of the matrix $\boldsymbol{B}$ using the conjugate gradient method without preconditioners. In all cases, both the condition number and the CPU time are much worse than those in Tables 2 and 3 , 
TABLE 3. Condition numbers of the Schwarz operator using $\rho_{3,2}(r)$ as $\mathrm{RBF}$

\begin{tabular}{|c|c|c|c|c|c|c|c|c|c|c|c|}
\hline$N$ & $q_{X}$ & $\cos \alpha$ & $\cos \beta$ & $M_{1}$ & $M_{2}$ & $J$ & $\lambda_{\min }$ & $\lambda_{\max }$ & $\kappa(P)$ & CPU & Iter. \\
\hline 10443 & $\frac{\pi}{240}$ & 0.99 & -0.52 & 140 & 367 & 420 & 0.023 & 49.02 & 2171.42 & 100.3 & 137 \\
& & 0.98 & -0.70 & 86 & 205 & 223 & 0.107 & 31.83 & 296.84 & 55.9 & 71 \\
& & 0.97 & -0.64 & 56 & 131 & 138 & 0.059 & 23.02 & 391.40 & 64.9 & 70 \\
& & 0.95 & -0.63 & 43 & 91 & 94 & 0.186 & 18.57 & 99.91 & 62.5 & 44 \\
& & 0.85 & -0.93 & 19 & 30 & 31 & 0.862 & 10.50 & 12.19 & 127.6 & 19 \\
& & 0.60 & -0.86 & 13 & 14 & 15 & 1.121 & 9.30 & 8.29 & 836.4 & 16 \\
\hline 13775 & $\frac{\pi}{280}$ & 0.99 & -0.40 & 141 & 374 & 429 & 0.031 & 49.04 & 1592.04 & 150.7 & 112 \\
& & 0.98 & -0.51 & 85 & 206 & 226 & 0.090 & 31.35 & 348.66 & 96.7 & 63 \\
& & 0.97 & -0.57 & 56 & 132 & 140 & 0.199 & 22.93 & 115.35 & 83.6 & 46 \\
& & 0.95 & -0.62 & 44 & 93 & 96 & 0.290 & 19.24 & 66.25 & 112.3 & 38 \\
& & 0.85 & -0.89 & 21 & 33 & 34 & 0.815 & 11.03 & 13.55 & 322.5 & 20 \\
& & 0.60 & -0.77 & 11 & 12 & 13 & 0.961 & 8.49 & 8.83 & 1732.7 & 17 \\
\hline 17078 & $\frac{\pi}{320}$ & 0.99 & -0.44 & 148 & 387 & 442 & 0.044 & 51.27 & 1153.38 & 167.6 & 101 \\
& & 0.98 & -0.71 & 85 & 207 & 227 & 0.188 & 32.72 & 173.62 & 121.0 & 60 \\
& & 0.97 & -0.52 & 59 & 137 & 146 & 0.134 & 24.58 & 182.98 & 138.3 & 53 \\
& & 0.95 & -0.81 & 44 & 93 & 96 & 0.335 & 18.66 & 55.69 & 171.1 & 36 \\
& & 0.85 & -0.92 & 20 & 31 & 32 & 0.709 & 10.81 & 15.24 & 614.3 & 21 \\
& & 0.60 & -0.79 & 11 & 12 & 13 & 0.805 & 8.51 & 10.58 & 3549.5 & 18 \\
\hline
\end{tabular}

TABLE 4. Conjugate gradient method without preconditioners

\begin{tabular}{|c|c|c|c|c|c|c|}
\hline$N$ & $q_{X}$ & $\lambda_{\min }$ & $\lambda_{\max }$ & $\kappa(\boldsymbol{B})$ & CPU & Iter. \\
\hline 10443 & $\frac{\pi}{240}$ & $0.2585 \mathrm{E}-03$ & $0.6767 \mathrm{E}+04$ & $0.2618 \mathrm{E}+08$ & 2204.98 & 3512 \\
13775 & $\frac{\pi}{280}$ & $0.3362 \mathrm{E}-04$ & $0.8082 \mathrm{E}+03$ & $0.2404 \mathrm{E}+08$ & 2802.73 & 2845 \\
17078 & $\frac{\pi}{320}$ & $0.4090 \mathrm{E}-04$ & $0.1022 \mathrm{E}+04$ & $0.2499 \mathrm{E}+08$ & 4545.70 & 2981 \\
\hline
\end{tabular}

We also compute the discrete maximum errors and $\ell_{2}$ errors over a set $\mathcal{G}=\left\{x_{g}\right\}$ of 19075 points over $S^{2}$ by

$$
\operatorname{err}_{\infty}=\max _{x_{g} \in \mathcal{G}}\left|u_{X}\left(x_{g}\right)-u\left(x_{g}\right)\right|
$$

and

$$
\operatorname{err}_{2}=\frac{1}{|\mathcal{G}|}\left(\sum_{x_{g} \in \mathcal{G}}\left|u\left(x_{g}\right)-u_{X}\left(x_{g}\right)\right|^{2}\right)^{1 / 2}
$$

where $|\mathcal{G}|=19075$ is the cardinality of $\mathcal{G}$. Tables $[5$ and $[$ summarize the computed results. 
TABLE 5. Errors using $\rho_{3,3}(r)$ as RBF

\begin{tabular}{|c|c|c|c|c|c|c|c|}
\hline$N$ & $\cos \alpha$ & $\cos \beta$ & $M_{1}$ & $M_{2}$ & $J$ & err $_{\infty}$ & err $_{2}$ \\
\hline 10443 & 0.99 & -0.52 & 140 & 367 & 420 & $8.4101 \mathrm{e}-04$ & $4.3393 \mathrm{e}-07$ \\
& 0.98 & -0.70 & 86 & 205 & 223 & $8.4257 \mathrm{e}-04$ & $4.3417 \mathrm{e}-07$ \\
& 0.97 & -0.64 & 56 & 131 & 138 & $8.4283 \mathrm{e}-04$ & $4.3421 \mathrm{e}-07$ \\
& 0.95 & -0.63 & 43 & 91 & 94 & $8.4294 \mathrm{e}-04$ & $4.3421 \mathrm{e}-07$ \\
& 0.85 & -0.93 & 19 & 30 & 31 & $8.4320 \mathrm{e}-04$ & $4.3420 \mathrm{e}-07$ \\
& 0.75 & -0.90 & 14 & 18 & 19 & $8.4336 \mathrm{e}-04$ & $4.3420 \mathrm{e}-07$ \\
\hline 13775 & 0.99 & -0.40 & 141 & 374 & 429 & $7.9116 \mathrm{e}-04$ & $3.9836 \mathrm{e}-07$ \\
& 0.98 & -0.51 & 85 & 206 & 226 & $7.9160 \mathrm{e}-04$ & $3.9836 \mathrm{e}-07$ \\
& 0.97 & -0.57 & 56 & 132 & 140 & $7.9139 \mathrm{e}-04$ & $3.9833 \mathrm{e}-07$ \\
& 0.95 & -0.62 & 44 & 93 & 96 & $7.9172 \mathrm{e}-04$ & $3.9838 \mathrm{e}-07$ \\
& 0.85 & -0.89 & 21 & 33 & 34 & $7.9103 \mathrm{e}-04$ & $3.9834 \mathrm{e}-07$ \\
& 0.75 & -0.91 & 15 & 18 & 19 & $7.9144 \mathrm{e}-04$ & $3.9836 \mathrm{e}-07$ \\
\hline 17078 & 0.99 & -0.44 & 148 & 387 & 442 & $7.0749 \mathrm{e}-04$ & $3.5143 \mathrm{e}-07$ \\
& 0.98 & -0.71 & 85 & 207 & 227 & $7.0767 \mathrm{e}-04$ & $3.5141 \mathrm{e}-07$ \\
& 0.97 & -0.52 & 59 & 137 & 146 & $7.0722 \mathrm{e}-04$ & $3.5151 \mathrm{e}-07$ \\
& 0.95 & -0.81 & 44 & 93 & 96 & $7.0739 \mathrm{e}-04$ & $3.5149 \mathrm{e}-07$ \\
& 0.85 & -0.92 & 20 & 31 & 32 & $7.0728 \mathrm{e}-04$ & $3.5144 \mathrm{e}-07$ \\
& 0.75 & -0.91 & 15 & 18 & 19 & $7.0796 \mathrm{e}-04$ & $3.5147 \mathrm{e}-07$ \\
\hline
\end{tabular}

TABLE 6. Errors using $\rho_{3,2}(r)$ as RBF

\begin{tabular}{|c|c|c|c|c|c|c|c|}
\hline$N$ & $\cos \alpha$ & $\cos \beta$ & $M_{1}$ & $M_{2}$ & $J$ & err $_{\infty}$ & rrr $_{2}$ \\
\hline 10443 & 0.99 & -0.52 & 140 & 367 & 420 & $1.0816 \mathrm{e}-03$ & $4.5627 \mathrm{e}-07$ \\
& 0.98 & -0.70 & 86 & 205 & 223 & $1.0802 \mathrm{e}-03$ & $4.5629 \mathrm{e}-07$ \\
& 0.97 & -0.64 & 56 & 131 & 138 & $1.0798 \mathrm{e}-03$ & $4.5628 \mathrm{e}-07$ \\
& 0.95 & -0.63 & 43 & 91 & 94 & $1.0796 \mathrm{e}-03$ & $4.5624 \mathrm{e}-07$ \\
& 0.85 & -0.93 & 19 & 30 & 31 & $1.0795 \mathrm{e}-03$ & $4.5621 \mathrm{e}-07$ \\
& 0.75 & -0.90 & 14 & 18 & 19 & $1.0794 \mathrm{e}-03$ & $4.5623 \mathrm{e}-07$ \\
\hline 13775 & 0.99 & -0.40 & 141 & 374 & 429 & $1.0601 \mathrm{e}-03$ & $4.3020 \mathrm{e}-07$ \\
& 0.98 & -0.51 & 85 & 206 & 226 & $1.0606 \mathrm{e}-03$ & $4.3021 \mathrm{e}-07$ \\
& 0.97 & -0.57 & 56 & 132 & 140 & $1.0603 \mathrm{e}-03$ & $4.3013 \mathrm{e}-07$ \\
& 0.95 & -0.62 & 44 & 93 & 96 & $1.0606 \mathrm{e}-03$ & $4.3020 \mathrm{e}-07$ \\
& 0.85 & -0.89 & 21 & 33 & 34 & $1.0605 \mathrm{e}-03$ & $4.3016 \mathrm{e}-07$ \\
& 0.75 & -0.91 & 15 & 18 & 19 & $1.0610 \mathrm{e}-03$ & $4.3019 \mathrm{e}-07$ \\
\hline 17078 & 0.99 & -0.44 & 148 & 387 & 442 & $9.6261 \mathrm{e}-04$ & $3.7418 \mathrm{e}-07$ \\
& 0.98 & -0.71 & 85 & 207 & 227 & $9.6174 \mathrm{e}-04$ & $3.7406 \mathrm{e}-07$ \\
& 0.97 & -0.52 & 59 & 137 & 146 & $9.6151 \mathrm{e}-04$ & $3.7400 \mathrm{e}-07$ \\
& 0.95 & -0.81 & 44 & 93 & 96 & $9.6209 \mathrm{e}-04$ & $3.7400 \mathrm{e}-07$ \\
& 0.85 & -0.92 & 20 & 31 & 32 & $9.6185 \mathrm{e}-04$ & $3.7400 \mathrm{e}-07$ \\
& 0.75 & -0.91 & 15 & 18 & 19 & $9.6209 \mathrm{e}-04$ & $3.7406 \mathrm{e}-07$ \\
\hline
\end{tabular}




\section{ACKNOWLEDGMENTS}

The support of the Australian Research Council under its Centres of Excellence program is gratefully acknowledged. The authors thank Professor Holger Wendland for detecting an error in an earlier version of the paper.

\section{REFERENCES}

1. R. K. Beatson, W. A. Light, S. Billings, Fast solution of the radial basis function interpolation equations: Domain decomposition methods. SIAM J. Sci. Comput. 22 (2000), 1717-1740. MR 1813294 (2001k:65025)

2. B. Bollobás, Modern graph theory, Springer-Verlag, New York, 1998. MR1633290|(99h:05001)

3. D. Chen, V. A. Menegatto, X. Sun, A necessary and sufficient condition for strictly positive definite functions on spheres. Proc. Amer. Math. Soc. 131 (2003), 2733-2740. MR1974330 (2004d:43007)

4. I. Halperin, The product of projection operators. Acta Sci. Math. (Szeged), 23 (1962), 96-99. MR0141978 (25:5373)

5. K. Hesse, Domain decomposition methods in multiscale geopotential determination from SST and $S G G$, Ph.D. thesis, University of Kaiserslautern, Germany, 2002.

6. S. Hubbert, T. M. Morton, A Duchon framework for the sphere, J. Approx. Theory 129 (2004), 28-57. MR2070179 (2005h:41047)

7. K. Jetter, J. Stöckler, J. D. Ward, Error estimates for scattered data interpolation, Math. Comp. 68 (1999), 743-747. MR1642746 (99i:41032)

8. Q. T. Le Gia, Galerkin approximation for elliptic PDEs on spheres, J. Approx. Theory 130 (2004), 125-149. MR2100699 (2005h:65215)

9. Q. T. Le Gia, F. J. Narcowich, J. D. Ward, H. Wendland, Continuous and discrete leastsquares approximation by radial basis functions on spheres. J. Approx. Theory 143 (2006), 124-133. MR2271729 (2007k:41078)

10. R. A. Langel, W. J. Hinze, The magnetic field of the earth's lithosphere, the satellite perspective, Cambridge University Press, Cambridge, 1998.

11. J. Levesley, Z. Luo, X. Sun, Norm estimates of interpolation matrices and their inverses associated with strictly positive definite functions. Proc. Amer. Math. Soc. 127 (1999), $2127-$ 2134. MR $1476145(99 \mathrm{j}: 41005)$

12. J. L. Lions, E. Magenes, Non-homogeneous boundary value problems and applications, Vol. I. Grundlehren der mathematischen Wissenschaften, Band 181, Springer-Verlag, New York, 1972. MR0350177 (50:2670)

13. T. M. Morton, M. Neamtu, Error bounds for solving pseudodifferential equations on spheres by collocation with zonal kernels. J. Approx. Theory 114 (2002), 242-268. MR1883408 (2002k:65200)

14. C. Müller, Spherical harmonics, Lecture Notes in Mathematics, Vol. 17. Springer, Berlin, 1966. MR0199449 (33:7593)

15. F. J. Narcowich, J. D. Ward, Scattered data interpolation on spheres: Error estimates and locally supported basis functions. SIAM J. Math. Anal. 33 (2002), 1393-1410. MR.1920637 (2003j:41021)

16. F. J. Narcowich, J. D. Ward, H. Wendland, Sobolev bounds on functions with scattered zeros, with applications to radial basis function surface fitting. Math. Comp. 74 (2004), 743-763. MR2114646 (2005k:41051)

17. S. V. Nepomnyaschikh, Domain decomposition and Schwarz methods in a subspace for approximate solution of elliptic boundary value problems. Ph.D. thesis, Computing Center of the Siberian Branch of the USSR Academy of Sciences, Novosibirsk, USSR, 1986.

18. J. von Neumann. Functional Operators. Vol. II. The Geometry of Orthogonal Spaces, Annals of Mathematics Studies, no. 22. Princeton University Press, Princeton, N. J., 1950. This is a reprint of mimeographed lecture notes first distributed in 1933. MR0034514 (11:599e)

19. J. G. Ratcliffe, Foundations of hyperbolic manifolds, Springer, New York, 1994. MR1299730 (95j:57011)

20. I. J. Schoenberg, Positive definite function on spheres. Duke Math. J. 9 (1942), 96-108. MR $0005922(3: 232 c)$ 
21. K. T. Smith, D. C. Solmon, and S. L. Wagner. Practical and mathematical aspects of the problem of reconstructing objects from radiographs. Bull. Amer. Math. Soc., 83 (1977), 12271270. MR0490032 (58:9394a)

22. K. T. Smith, D. C. Solmon, and S. L. Wagner. Addendum to: "Practical and mathematical aspects of the problem of reconstructing objects from radiographs" (Bull. Amer. Math. Soc. 83 (1977), no. 6, 1227-1270). Bull. Amer. Math. Soc., 84 (1978), 691. MR0490033 (58:9394b)

23. A. Toselli, O. B. Widlund, Domain decomposition methods-algorithms and theory, SpringerVerlag, Berlin 2005. MR2104179 (2005g:65006)

24. T. Tran, Overlapping additive Schwarz preconditioners for boundary element methods. J. Integral Equations Appl. 12 (2000), 177-207. MR.1771517 (2001i:65136)

25. T. Tran, E. P. Stephan, Additive Schwarz methods for the h-version boundary element method, Appl. Anal. 60 (1996), 63-84. MR1623443 (99a:65169)

26. T. Tran, E. P. Stephan, An Overlapping Additive Schwarz Preconditioner for Boundary Element Approximations to the Laplace Screen and Lamé Crack Problems, J. Numer. Math. 12 (2004), 311-330. MR2107935 (2005i:65198)

27. H. Wendland, Piecewise polynomial, positive definite and compactly supported radial functions of minimal degree., Adv. in Comp. Math. 4 (1995), 389-396. MR1366510 (96h:41025)

28. H. Wendland, Error estimates for interpolation by compactly supported radial basis functions of minimal degree. J. Approx. Theory 93 (1998), 258-272. MR.1616781 (99g:65015)

29. H. Wendland, Scattered data approximation, Cambridge University Press, Cambridge, 2005. MR2131724 (2006i:41002)

30. O. B. Widlund, Optimal iterative refinement methods, T. F. Chan, R. Glowinsky, J. Périaux, O. B. Widlund (eds.), Proceedings of the 2nd international symposium on domain decomposition methods for partial differential equations. SIAM, Philadelphia, 1989. MR992008 (90m:65205)

31. Y. Xu, E. W. Cheney, Strictly positive definite functions on spheres. Proc. Amer. Math. Soc. 116 (1992), 977-981. MR1096214 (93b:43005)

School of Mathematics and Statistics, The University of New South Wales, Sydney 2052, Australia

E-mail address: qlegia@maths.unsw.edu.au

School of Mathematics and Statistics, The University of New South Wales, Sydney 2052, Australia

E-mail address: I.Sloan@unsw.edu.au

School of Mathematics and Statistics, The University of New South Wales, Sydney 2052, Australia

E-mail address: Thanh.Tran@unsw.edu.au 